\title{
Monte Carlo Simulations of Grain Growth in Metals
}

\author{
Sven K. Esche \\ Stevens Institute of Technology \\ USA
}

\section{Introduction}

The application of the Monte Carlo (MC) method to simulate the grain growth in metals originates from Potts' model for magnetic domain evolution (Potts, 1952), which generalized the two-state spin up or spin down ferromagnetic Ising model to systems with arbitrary spin degeneracy. Subsequently, the so-called n-fold method for expediting simulations of the time evolution of systems was developed (Bortz et al., 1975). Anderson and his co-workers were the first to introduce the Potts model into grain growth simulations, applying this method to model the grain growth kinetics (Anderson et al., 1984), grain size distribution and topology (Srolovitz et al., 1984a), influence of particle dispersions (Srolovitz et al., 1984b), anisotropic grain boundary energies (Grest et al., 1985) as well as abnormal grain growth (Srolovitz et al., 1985; Rollett et al., 1989; Rollett \& Mullins, 1996). By incorporating specific elements corresponding to various microstructural processes into the basic algorithm, the MC method has been adapted to model for instance grain growth in twophase materials (Holm et al., 1993) and composites (Miodownik et al., 2000), abnormal grain growth (Lee at al., 2000, Messina et al., 2001; Ivasishin et al., 2004), static recrystallization (Srolovitz et al., 1986; Srolovitz et al., 1988; Rollett et al., 1992a, Rollett \& Raabe, 2001; Song \& Rettenmayr, 2002)), dynamic recrystallization (Peczak, 1995; Rollett et al., 1992b) and sintering (Hassold, et al., 1990; Chen et al., 1990, Matsubara, 1999), and it has been demonstrated that such MC simulations are capable of reproducing the essential features of these microstructural phenomena. Nowadays, the MC method is often preferred to deterministic methods such as cellular automaton (Geiger et al., 2001) and phase-field models (Tikare et al., 1998) at the mesoscopic level, mainly due to its inherent simplicity and flexibility. More recently, the MC method has also been employed to predict the final microstructures in engineering applications (Yang et al., 2000; Yu \& Esche, 2005).

For quite some time, numerous efforts geared toward improving the accuracy and efficiency of the conventional MC method have been reported in the literature (Radhakrishnan \& Zacharia, 1995; Song \& Liu, 1998, Yu \& Esche, 2003a), aiming at providing the foundation for the application of the MC method in engineering practice. Various modifications of the conventional Monte Carlo (CMC) algorithm have been reported. For instance, an increase in processing speed of up to two orders of magnitude compared with the CMC algorithm were achieved in grain growth simulations by employing a modified MC algorithm (Yu \& Esche, 2003a). Furthermore, this modified algorithm also led to an improved accuracy of the predicted grain growth exponent in the kinetic equations, particularly in small grain size 
regimes (i.e., for grain sizes ranging from 1 to 15 lattice spacing units). This improvement of the accuracy of the simulation results also facilitated new insights into the performance of MC Potts models for simulating the microstructure evolution (Yu \& Esche, 2003b, Yu \& Esche, 2003c).

The kinetics of normal grain growth is governed by the following equation (Atkinson, 1998)

$$
\langle R\rangle^{m}-\langle R\rangle_{0}^{m}=M t
$$

where $\left\langle\mathrm{R}>\right.$ is the average grain radius, $\langle\mathrm{R}\rangle_{0}$ is the initial average grain radius, $\mathrm{m}$ and $\mathrm{M}$ are constants and $t$ is the time. If in the analyzed time interval the initial grain radius $\langle R\rangle_{0}$ is negligible compared with $\langle\mathrm{R}\rangle$, then the grain growth kinetics can be simplified to (Louat, 1974)

$$
\langle R\rangle=K t^{n}=M^{n} t^{n}
$$

where $n=1 / m$ is the grain growth exponent.

Despite the fact that there is ample theoretical (Burke \& Turnbull, 1952; Mullins, 1956; Mullins \& Vinals, 1989), computational (Weiare \& Kermode, 1984) as well as experimental (Glazier et al., 1987) evidence that the kinetic law is parabolic (i.e. $\mathrm{n}=0.5$ ), a substantial number of research results obtained from theoretical work (Rhines et al., 1974), computer simulations (Anderson et al., 1984) and physical grain growth experiments (Bolling \& Winegard, 1958; Anderson et al., 1984) contradict the parabolic kinetic law. In physical experiments, the deviation from the parabolic kinetics can be explained by the varying grain boundary mobility and energy of the material samples or by the presence of a small number of second-phase particles in the material samples (Humphreys \& Hatherly, 1996). However, the results of computer simulations with ideal (i.e., isotropic, single-phase) materials have also exhibited the deviation from the parabolic kinetic law. Even though theoretically the self-similarity of the microstructure evolution is a sufficient condition for the occurrence of the parabolic kinetic law in the curvature-driven grain growth process, some results showed that two-dimensional (2D) normal grain growth simulations of single-phase systems employing the MC Potts model result in a significantly lower exponent of $n=0.41$ in the small grain size regimes (Anderson et al., 1984; Grest et al., 1988), despite the fact that selfsimilarity was observed. The grain growth exponent was found to asymptotically approach $\mathrm{n}=0.5$ at the later simulation stages when using large lattice systems (up to $1000 \times 1000$ lattice points) with large final grain sizes (Grest et al., 1988) while in the small grain size regimes, it was believed that an unphysical finite-size effect is likely to dominate (Anderson et al., 1989). Furthermore, it was also demonstrated that normal grain growth is modeled almost precisely (i.e. with an exponent of $n=0.502$ ) if small clusters are excluded in calculations of the microstructure statistics (Ivasishin et al., 2003). The lower exponent $n$ observed when including these small clusters was attributed to the presence of two competing length scales in the simulation, namely the mean grain size $\langle\mathrm{R}>$ and the lattice spacing $\mathrm{a}_{0}$ (Kumar et al., 1987; Beenakker, 1988), and thus the effect of the finite lattice spacing and the self-similarity of the grain growth can be expected only in the limit when $<\mathrm{R}>>>\mathrm{a}_{0}$. It should be noted though that this unphysical finite-size effect is different from lattice type effects. Some lattice types (e.g. square lattices with four nearest neighbors) exhibit a strong lattice anisotropy, which in turn may cause the grain growth simulated using these types of lattices to stop artificially. A comprehensive review of the effects of 
various neighborhood types on the kinetics of grain growth can be found elsewhere (Raabe, 2002). Other researchers attributed the low grain growth exponent in the small grain size regimes to the smaller probability of successful reorientation attempts (Holm, 1992; Radhakrishnan \& Zacharia, 1995; Song et al., 1998). Therefore, the grain growth exponent varies with the grain size and asymptotically approaches a value close to 0.5 at a grain size of 15.8 when using modified MC algorithms (Radhakrishnan \& Zacharia, 1995; Mehnert \& Klimanek, 1997). In addition, it was argued that in the presence of second-phase particles the simulation results are significantly affected by Zener pinning (Mehnert \& Klimanek, 1996; Kad \& Hazzledine, 1997; Soucail et al., 1999), and furthermore, three-dimensional (rather than cross-sectional) grain size distributions should be used in some cases (Xiaoyan et al., 2000).

In view of the phenomena discussed above, large lattice systems have to be used in order to preserve a sufficient number of grains for statistical analysis since the data in small grain size regimes have to be discarded (Grest el al., 1988; Holm et al., 2001; Miodownik, 2002). However, large lattice sizes are undesirable because they hamper the application of the MC method in engineering practice. A modified MC algorithm developed later was shown to generate $\mathrm{n} \approx 0.5$ for both small and large grain sizes (Yu \& Esche, 2003a).

In this chapter, first some theories on the self-similarity and kinetics of normal grain growth of isotropic single-phase materials are briefly reviewed. Then, both the two-dimensional $\mathrm{CMC}$ algorithm and various modifications and improvements introduced later are described, followed by a discussion of the extension of the MC method to three-dimensional problems. Finally, multi-scale approaches for microstructure predication are briefly introduced.

\section{Theories on self-similarity and kinetics of normal grain growth}

\subsection{Burke and Turnbull's analysis}

For a spherical boundary with curvature $\kappa$, the velocity $v$ of the boundary movement toward its center of curvature is (Burke \& Turnbull, 1952)

$$
v=M_{0} \gamma \kappa
$$

where $\mathrm{M}_{0}$ is the mobility and $\gamma$ is the grain boundary energy. Assuming that for a grain of radius $R$ the radius of curvature $R_{k}$ of each spherical boundary face is proportional to $R$, then the time rate of change of $R$ is equal to the velocity $v$ of the boundary movement. Therefore,

$$
\begin{gathered}
R_{\kappa}=\frac{2}{\kappa} \sim R \\
\frac{d R}{d t}=\frac{2 M_{0} \gamma C}{R}
\end{gathered}
$$

where $t$ is the time and $C$ is a dimensionless constant. Moreover, let us assume that the average grain radius $<R>$ evolves with the same functional relationship as any arbitrary $R$, which is equivalent to assuming structural self-similarity. Then, for an isotropic system (for which $\mathrm{M}_{0}$ and $\gamma$ are constants), substituting $<\mathrm{R}>$ for $\mathrm{R}$ into Eq. (5) and integrating this equation yields: 


$$
\langle R\rangle^{2}-\langle R\rangle_{0}^{2}=2 M_{0} \gamma C t
$$

However, it should be noted that in realty the entire grain boundary rarely has a uniform curvature.

\subsection{Von Neumann's law}

Von Neumann's law (Von Neumann, 1952; Mullins, 1956) is based on topological considerations. In the two-dimensional case, the rate of change of the area of a grain $\mathrm{G}$ is

$$
\frac{d A}{d t}=-\int_{G} v d s
$$

where $\mathrm{A}$ is the grain area, $\mathrm{s}$ is the length of the grain boundary and the integration is carried out over all grain boundary segments between grain corners where three or more grains meet. Evaluating the integral of the grain boundary curvature $\kappa$ around a two-dimensional grain $\mathrm{G}$ results in

$$
\int_{G} \kappa d s=2 \pi-\sum_{i=1}^{n_{\theta}} \theta_{i}
$$

where $\kappa$ is positive toward the center of the grain and the $\theta_{i}$ are the complements of the interior angles at each of the $n_{e}$ grain corners. Let us now assume that the only stable grain corner in an isotropic grain system is the three-grain junction (referred to as tri-junction) and furthermore that all grain corner angles at tri-junctions are equal to $2 \pi / 3$ in order to balance the interfacial energy. Thus, $\theta_{i}=\pi / 3$ and for isotropic systems, the following equation can be deduced:

$$
\frac{d A}{d t}=-M_{0} \gamma \int_{G} \kappa d s=-\frac{1}{3} \pi M_{0} \gamma\left(6-n_{e}\right)
$$

If grain growth is assumed to exhibit structural self-similarity, then by integrating the above equation, the average grain area $\langle A>$ can be shown to be proportional to the time $t$, and thus, the parabolic grain growth law is obtained.

\subsection{Mullins' self-similarity theory}

Consider a system with total volume $\mathrm{V}$ that contains a large number of particles $\mathrm{N}$. Then, the mean particle volume $\bar{V}$ may be written in the form (Mullins, 1986):

$$
\bar{V}=\frac{V}{N}
$$

Differentiating the above equation with respect to time $t$ results in:

$$
\frac{d \bar{V}}{d t}=-\frac{\bar{V}}{N} \frac{d N}{d t}
$$

Using the continuity equation, it can be shown that (Mullins, 1986)

$$
\frac{d N}{d t}=\lim _{V_{0} \rightarrow 0} \int_{-\infty}^{0} \dot{V} \rho\left(V_{0}, \dot{V}, t\right) d \dot{V}
$$


where $\mathrm{V}$ is the volume of individual particles with $\dot{V}=d V / d t, \mathrm{~V}_{0}$ is the volume at $\mathrm{t}=0$ and $\rho$ is the probability density function. Therefore,

$$
\begin{aligned}
& \frac{d \bar{V}}{d t}=-\bar{V} \lim _{V_{0} \rightarrow 0} \int_{-\infty}^{0} \dot{V} f\left(V_{0}, \dot{V}, t\right) d \dot{V} \\
& f\left(V_{0}, \dot{V}, t\right)=\frac{1}{N} \rho\left(V_{0}, \dot{V}, t\right)
\end{aligned}
$$

where $\mathrm{f}$ is referred to as normalized density function. If the statistical self-similarity (SSS) holds (i.e. if $\mathrm{f}$ is time independent), then the following equation can be derived

$$
[\bar{V}(t)]^{1-a}-[\bar{V}(0)]^{1-a}=(1-a) C t
$$

where $C$ is a constant and $\alpha$ is a constant that depends on the system under consideration. For isothermal grain growth, $\alpha$ is found to be $1 / 3$, and since $<\mathrm{R}>$ is proportional to $\bar{V}^{1 / 3}$, the parabolic grain growth law is therefore obtained again. It should be noted that, as long as the SSS holds, this result applies to both isotropic and anisotropic grain growth.

\section{Conventional Monte Carlo method for grain growth simulations}

In the conventional MC method for simulating two-dimensional (2D) isotropic normal grain growth of single-phase materials (Anderson et al., 1984; Holm, 1992), a continuum microstructure is mapped onto a 2D lattice, whereby the most commonly used lattice type is the triangular lattice with six nearest neighbors (Rollett, 1997).

First, an integer number $S_{i}$ between 1 and $Q$ is assigned to each lattice site (where $Q$ represents the total number of grain orientations in the system). This process is known as the initialization of the lattice. Then, the MC algorithm iteratively transforms the lattice in a procedure comprising the following four-steps: (i) random selection of a lattice site, (ii) assignment of a new orientation number to this site, which is selected randomly from all the other Q-1 orientation numbers in the system, and (iii) calculation of the net change of the system energy $\Delta \mathrm{E}$ due to the reorientation at the selected lattice site. The total energy $\mathrm{E}$ is usually defined as

$$
E=J_{g b} \sum_{\langle i j\rangle}\left(1-\delta_{S_{i} S_{j}}\right)
$$

where $\mathrm{J}_{\mathrm{gb}}$ is the grain boundary energy scale, $<\mathrm{ij}>$ is the nearest neighbor site pair and $\delta_{\mathrm{SiSj}}$ is the Kronecker delta function. Note that in certain cases it is necessary to include the second nearest neighbors into the energy calculations (Anderson et al., 1989, Holm et al., 1991). Finally, in step (iv) of the MC algorithm, the reorientation attempt is accepted with a probability $\mathrm{p}$

$$
p(\Delta E)=\left\{\begin{array}{ccc}
1 & \text { if } & \Delta E \leq 0 \\
\exp \left(-\frac{\Delta E}{k T}\right) & \text { if } & \Delta E>0
\end{array}\right.
$$


where $\mathrm{k}$ is the Boltzmann constant and $\mathrm{T}$ is the simulation temperature (not the physical temperature), which is introduced to avoid stagnation of the lattice evolution in some cases (Holm et al., 1991). If $\Delta \mathrm{E}$ is non-positive (i.e. $\Delta \mathrm{E} \leq 0$ ), the attempted reorientation is accepted. If $\Delta \mathrm{E}>0$, a random number between 0 and 1 is generated and the attempted reorientation is accepted if this random number is smaller than the probability $p$, and otherwise, the old orientation of the site is recovered. In practice, a zero temperature probability is often adopted (Holm et al., 1991):

$$
p(\Delta E)=\left\{\begin{array}{lll}
1 & \text { if } & \Delta E \leq 0 \\
0 & \text { if } & \Delta E>0
\end{array}\right.
$$

In the MC method, the temporal evolution of the simulated physical process is modeled in terms of a simulation time scale referred to as Monte Carlo Steps (MCS). Each MCS comprises $\mathrm{N}$ reorientation attempts, where $\mathrm{N}$ is the total number of lattice sites in the system. Furthermore, the MCS is assumed to be linearly related with the physical time through a jump frequency that depends on the physical temperature (Safran et al., 1983, Raabe, 2000).

In the context of the MC method, a grain is defined as a set of adjacent lattice sites that are associated with identical orientation numbers. The grain boundaries are formed by the interface lines between site pairs of unlike orientation. Then, the area A of a grain is defined as the number of lattice sites within one grain, and the radius $\mathrm{R}$ of a grain is commonly defined as the square root of the corresponding grain area (Holm, 1992). Lastly, the mean grain radius $<\mathrm{R}>$ is defined as the square root of the total number of lattice sites divided by the total number of grains.

It should be pointed out that the underlying algorithm of the conventional MC method is stochastic in nature and thus does not reflect the physical principles inherent in the grain growth process. Its major physical and numerical shortcomings are its inability to predict the theoretically expected grain growth exponent $n$ in the small grain size regime, the possible occurrence of unrealistic nucleation events and its general numerical inefficiency.

The normal grain growth is curvature driven and obeys a power law of the form of Eq. 1 (Hillert, 1965, Louat, 1974). For large grain radii $\langle\mathrm{R}\rangle$, this relationship simplifies to the form of Eq. 2. There is ample theoretical (Burke \& Turnbull, 1952; Mullins \& Vinals, 1989), computational (Weiare \& Kermode, 1984; Grest et al., 1988) and experimental (Weiare \& Kermode, 1983; Glazier et al., 1987) evidence that the grain grown exponent equals $n=0.5$. Nevertheless, a lower grain growth exponent is often reported for the earlier stages of simulations with the conventional MC method (e.g. $\mathrm{n}=0.41$, Anderson et al., 1984), which contradicts the value of $n=0.5$ that is theoretically expected under the assumption of selfsimilarity. The lower than expected grain growth exponent is usually said to be the result of the continuously decreasing boundary mobility in the early transition stage of simulated grain growth, whereas in the later simulation stages, the grain growth kinetics stabilizes (Holm, 1992; Radhakrishnan \& Zacharia, 1995; Song et al., 1998). Some other researchers opined that numerical finite size effects are likely to dominate when the grain size is small (Grest at al., 1988; Anderson et al., 1989). Others suggested that the lower than expected grain growth exponent observed in the small grain size regime of the simulation is due to the mean grain size $<\mathrm{R}>$ and the lattice spacing $\mathrm{a}_{0}$ representing two competing length scales (Kumar et al., 1987; Beenakker, 1988). Only if $\left\langle\mathrm{R}>>>\mathrm{a}_{0}\right.$ is the effect of the finite lattice spacing $\mathrm{a}_{0}$ negligible and the grain growth expected to be truly self-similar. 
The second deficiency of the conventional MC algorithm is the occurrence of grain nucleation by reorientation in some instances, for example in simulations with non-zero temperature and in simulations of anisotropic grain growth. Of course, this nucleation phenomenon does not correlate with the actual physical grain growth process. In non-zero temperature simulations, a grain might nucleate at any lattice site due to the artificially introduced "thermal fluctuation", in spite of this nucleation causing an increase in the system energy. Also, if the grain boundary energy is anisotropic, a new grain with smaller grain boundary energy may be nucleated by reorientation alone - a phenomenon that in reality would be prevented based on thermodynamic considerations.

The third shortcoming of the conventional MC method is that it is very time consuming (Bortz et al., 1975; Song et al., 1998). This inefficiency is attributable mainly to the fact that the vast majority of attempted lattice point reorientations cause net energy increases, and therefore, the probability for energy-reducing reorientations leading to actual net grain growth is extremely small. In addition, for large two-dimensional (2D) or three-dimensional (3D) lattice systems and for large numbers of possible orientations $Q$, this situation even worsens progressively.

Scaling constitutes another important problem associated with the MC method since it does not involve any physical time or length parameters. The only time constant involved is the MCS, and the spacing of the simulation lattice represents the only length constant. Thus, in order to relate the MC simulation results to actual physical units, both time and length scaling is needed. While several scaling approaches for the MC method have been suggested, this issue is still far from being resolved (Raabe, 2000; Raabe, 2002; Janssens et al., 2007; Nosonovsky et al., 2009).

\section{Modified Monte Carlo algorithms for grain growth}

From a purely computational point of view, the n-fold method, which was originally proposed for the Ising model (Bortz et al., 1975), was later extended to zero-temperature grain growth simulations (Sahni et al., 1983). In the $n$-fold method, the lattice sites were separated into two types. The first type consists of those lattice sites for which any orientation switches are rejected because they increase the total energy in the system. The second type comprises those sites for which orientation switches to at least one of the other Q-1 orientations cause a reduction in the total energy. Later, the concept of site activity - a parameter that is calculated based on the orientations of the neighboring sites - was introduced (Holm, 1992). In this approach, a site of the second type is randomly selected with a frequency proportional to its activity, and a new orientation is selected randomly among the energetically favorable orientations. One additional random number is used to estimate the time elapsed (measured in MCS) between two successive reorientation attempts. This method ensures that every reorientation attempt is successful, and therefore it significantly increases the computational efficiency. However, there are four major shortcomings associated with the n-fold method. First, the algorithm is more complicated than other modifications of the MC method. Second, an additional random number is required that has no clear physical meaning. Third, this technique is only valuable and applied in the later stages of grain growth when most of the lattice sites are bulk sites and cannot be reoriented successfully. Thus, the grain growth kinetics obtained in the early stages of the simulation is not affected. Fourth, artificial grain nucleation and grain coalescence may still occur in some cases of non-zero temperature simulation or anisotropic 
grain boundary energies, and therefore this method may not be universally applicable without further modifications.

The early MC algorithms for modeling grain growth did not incorporate the physics of the process. Later, a modified MC method that considers the grain growth physics was suggested (Radhakrishnan \& Zacharia, 1995), although the resulting algorithm is similar to the n-fold method discussed above. It was argued that, since in reality grain growth occurs through atom migration from one grain to another across the corresponding grain boundary, an attempted reorientation should not be selected from all possible values of orientations but rather it should be restricted to one of the nearest neighbors' orientations. Therefore, it was proposed to generate a random number in the range between one and the total number of nearest neighbors of the lattice site under consideration. Based on this random number, the corresponding neighbor's orientation is assigned to the attempted lattice site for evaluation. This modification prevents artificial grain nucleation during grain growth and thus renders the MC model more realistic. Another recommendation was to select the new orientation amongst those of the unlike neighbors without weighting the selection by the number of neighbors that possess this new orientation as in the method above (Holm \& Battaile, 2001). This approach is equivalent to the n-fold method.

Furthermore, it was suggested that there is no physical meaning to randomly selecting lattice sites for reorientation (Song et al., 1998; Song \& Liu, 1998), and it was therefore proposed to evaluate them one by one in each MCS. By this approach, it is not only ensured that computing time is saved but also that all lattice sites are attempted for reorientation exactly once in each MCS.

The above modifications contributed to the resolution of the shortcomings associated with the conventional MC algorithm for grain growth simulations. However, they did not fully take the grain growth physics into account. Therefore, they generated grain growth exponents close to $\mathrm{n}=0.5$ only for very large average grain sizes $\langle R\rangle$, while the lower grain growth exponents typically obtained in the early simulation stages remained basically unchanged. This imperfect modeling may be attributable to the procedural algorithm design methodology employed. More recently, an algorithm that better reflects the physical behavior observed in nature was introduced ( $Y u$ \& Esche, 2003a). The accuracy and efficiency of an algorithm may be affected by its design methodology, whereby procedural and object-oriented approaches represent the two fundamental methodologies. The objectoriented design is preferable for modeling the static and dynamic behaviors of physical objects without using analytical equations of motion (Yu \& Esche, 2004). Note that the conventional MC algorithm for simulating the grain growth process was designed in a procedural fashion. While it was able to reproduce some essential process features with relative success, it still exhibited significant potential for improvements in accuracy and efficiency. The n-fold method discussed above significantly reduced the computational time compared with corresponding MC algorithms. However, it did neither conceptually change the conventional MC model nor improve the algorithm's accuracy. The algorithms proposed thereafter started to take into consideration the grain growth physics, and therefore, the efficiency, accuracy and capabilities of the MC simulations were greatly improved. However, these algorithms were still designed in a procedural manner and missed some essential physical considerations. Facilitated by the object-oriented algorithm design methodology, three modifications to the conventional MC algorithm were proposed ( $\mathrm{Yu} \&$ Esche, 2003a). 


\subsection{Modification I}

From the point of view of object-oriented programming, the lattice is composed of grains, and the lattice sites forming a grain are either at the boundary or in the interior of that grain. Grain growth occurs through grain boundary migration, or in other words, through the jump of atoms from one grain to another through the grain boundary. In the modified MC algorithm (Yu \& Esche, 2003a), when a lattice site is selected, its location is checked first. Interior sites do not migrate. Therefore, no reorientation is tried for such sites. If the selected site is located at a grain boundary, then the algorithm attempts a possible reorientation.

In order to save computing time, it was earlier proposed not to check for reorientation those lattice sites for which more than half of the neighboring lattice sites were of the same orientation (Sahni et al., 1983). This method does not exhibit any problems for isotropic systems. However, in systems with significant anisotropy of the grain boundary energies, this approach might under certain circumstances lead to the exclusion of viable, energyreducing reorientation attempts for those lattice sites. This method is not in accordance with the physics of grain boundary movements, and therefore it cannot be applied universally.

\subsection{Modification II}

Consider the grain structure in Figure 1. Suppose that the lattice site under consideration (circled) is located at the boundary of the grain with orientation 3 (grain 3 for short). The grains adjacent to this lattice site have orientations of either 1 or 2 . Furthermore, let us assume that grain 3 is currently shrinking. In that case, its boundary sites would either jump to the neighboring grains 1 or 2 or keep their current orientation, depending on whether the energy criterion for reorientation is satisfied or not. Thus, the new attempted orientation for the lattice site under consideration for reorientation should be 1 (or 2) if the corresponding site is adjacent to grain 1 (or 2) only or if the site is a triple point where three grains meet. In either case, the selection of the new orientation should be limited to those of the neighboring grains.

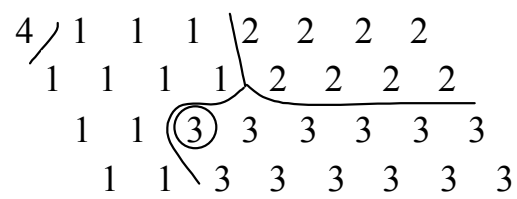

Fig. 1. Schematic of a grain structure

Modification II to the conventional MC algorithm ( $Y u$ \& Esche, 2003a) involves the generation of a random number in the range between 1 and $q$, where $q$ is the total number of nearest neighboring sites with orientations that differ from that of the attempted boundary site. Then, this random number is utilized to pick one of these neighbors and to assign its orientation to the attempted site. The improvement of this modification over the one proposed earlier (Radhakrishnan \& Zacharia, 1995) is that using Modification II, the circled site of grain 3 in Figure 1, for instance, would definitely jump to grain 1 and thus reduce the system energy. With the previously proposed modification, though, it would have jumped only with a probability of 4 out of 6 . Comparing Modification II with the prior suggestion (Holm \& Battaile, 2001), the selection of the new orientation is weighted by the total number of neighboring lattice sites with the same orientation. This is probably the case in a physical sense since grain growth represents a free energy reduction process that involves random 
movements to some degree, and very possibly, in the physical reality the free energy reduction occurs following the shortest possible path. Therefore, this modification provides the boundary sites with an optimal mobility and represents a more realistic grain growth model than the conventional MC algorithm as will be shown by the sample simulations below.

\subsection{Modification III}

When using the conventional MC algorithm, a subset of all lattice sites is considered multiple times for possible reorientation while some of the remaining sites are not attempted at all. Consider for instance a $200 \times 200$ lattice, introducing an index numbering the lattice sites from 1 to 40,000. There are two possible methods for generating the random number needed to select a lattice site whereby the random integer numbers can be generated using the function $\operatorname{rand}()$ in the standard $C$ library. Method 1 is $40,000 \times \operatorname{rand}() /$ RAND_MAX, where RAND_MAX $=32767$ is the maximum integer value that can be generated by rand(). Method 2 consists of producing two integer random numbers in order to obtain the coordinates of the lattice site to be selected for possible reorientation and then calculating the index of that site. In both methods, only a small fraction of all possible site indices occur exactly once in the 40,000 reorientation attempts while a significant portion do not occur at all (Yu \& Esche, 2003a). In each MCS, additional random numbers are also generated to select new orientations, but this is not likely to affect the uneven selection of lattice sites. As a consequence, the objective of attempting reorientation for every lattice site in each MCS cannot be met in a completely random fashion. Therefore, the microstructure does not evolve evenly in the entire lattice domain, and thus, the simulated grain growth kinetics is likely to be affected.

For that reason, another modification that eliminates the possibility of multiple selections of one lattice site within one MCS was introduced into the conventional MC algorithm (Yu \& Esche, 2003a). In this approach, the first lattice site is selected randomly from the total of $\mathrm{N}$ lattice sites in the system and evaluated for possible reorientation. Then, the second site is selected randomly only from the remaining N-1 lattice sites and then the next ones amongst the remaining $\mathrm{N}-2, \mathrm{~N}-3$ and so on. In this fashion, all sites are selected exactly once per MCS. Note that this modification still involves some element of randomness, which can be justified by two physical reasons. First, a grain boundary moves towards its center of curvature in a somewhat random fashion at smaller length scales. In fact, it was argued earlier that the motion of the grain boundary could be considered as sections of the boundary undergoing a random walk (Louat, 1974). Second, it would be desirable to update all boundary locations at the same time, but a method to achieve that objective without causing evolution stagnation has not been devised yet. On the other hand, when implementing Modification III, the movement of all grain boundaries during each MCS can be regarded as an approximation to the simultaneous movement of all boundaries at one physical time instant.

The above-mentioned earlier modification where all lattice sites are evaluated sequentially in each MCS (Song et al., 1998) might not correctly model the natural behavior of grain growth. In fact, when combining this approach with certain selection methods for the candidate orientations, it does not work at all. This can be demonstrated for instance by simulating the shrinkage of a hexagonal grain that is imbedded in an infinite matrix using a triangular lattice as shown in Figure 2. When combining this case with Modification II discussed above using zero-temperature probability and selecting reorientation sites one by 
one from left to right and top to bottom, the imbedded grain disappears immediately in a single MCS. Of course, this outcome does not reflect the correct grain shrinkage kinetics. Other conceivable artificial site selection algorithms may exhibit similar problems, and therefore, they would be undesirable if they lack underlying physical meaning.

$\begin{array}{cccccccccccc}0 & 0 & 0 & 0 & 0 & 0 & 0 & 0 & & & \\ 0 & 0 & 0 & 0 & 0 & 0 & 0 & 0 & & \\ 0 & 0 & 0 & 1 & 1 & 1 & 0 & 0 & & \\ & 0 & 0 & 1 & 1 & 1 & 1 & 0 & 0 & \\ & 0 & 0 & 1 & 1 & 1 & 0 & 0 & 0 & \\ & 0 & 0 & 0 & 0 & 0 & 0 & 0 & 0 \\ & 0 & 0 & 0 & 0 & 0 & 0 & 0 & 0\end{array}$

Fig. 2. A hexagonal grain (1) imbedded in a matrix (0)

\subsection{Simulation results}

As the simulation results and the discussion below demonstrate, the differences between the three modifications to the conventional MC algorithm and other modifications suggested previously are especially critical when considering small grain size regimes, where a large fraction of the lattice sites are located at grain boundaries. A detailed analysis of the performance of the three modifications to the conventional $\mathrm{MC}$ algorithm has been conducted by simulating grain shrinkage and grain growth (Yu \& Esche, 2003a), in which the resulting grain growth exponents $n$ obtained using least square regression analysis and the total number of MCS to achieve a certain mean grain radius $<\mathrm{R}>$ were compared with several preexisting algorithms. Furthermore, the variability of the simulation results with respect to different seeds used by the random number generator was analyzed.

\subsubsection{Simulation of grain shrinkage}

Simulating the kinetics of the shrinkage of an isolated circular grain that is imbedded in an infinite matrix is generally regarded as an effective means for testing the correctness and efficiency of a grain growth simulation procedure. In order to test the three modifications discussed above in the large grain size regime, the kinetics of the shrinking of a circular grain with an initial grain radius of $R_{0}=50$ was examined employing a $200 \times 200$ triangular lattice with six nearest neighbors. The following simulation procedures were compared:

1. Procedure 1: conventional MC algorithm

2. Procedure 2: MC algorithm with modification by Radhakrishnan and Zacharia, 1995

3. Procedure 3: MC algorithm with Modification II discussed above

4. Procedure 4: MC algorithm with Modifications II and III discussed above

The Ising Model (i.e. $Q=2$ ) was applied for the circular grain shrinkage simulation by Procedure 1 . Since Modification I saves about $20 \%$ of the computing time without affecting the lattice evolution, this modification was adopted for all four procedures.

Subsequently, the normal grain growth up to a mean grain radius of $<R>=25$ was simulated employing the four procedures listed above in conjunction with a $400 \times 400$ lattice. Lattices of this large size were used in order to prevent the simulated grain growth kinetics from getting distorted by lattice boundary effects. For Procedures 2, 3 and 4, the total number of possible orientations $Q$ can theoretically be equal to the total number of 
lattice sites $\mathrm{N}$ without seriously affecting the computing time. In this study, $\mathrm{Q}=10,000$ was employed for these three procedures, which is deemed large enough to render any possible artificially introduced grain coalescence insignificant. Note that for Procedure 1, the number of possible orientations has to be reduced significantly, and $Q=63$ was applied in this study. The affect of the choice of $Q$ on the simulation results when using Procedure 1 is well documented in the literature. Since the major concern is in the early stages of the simulations, the n-fold method was not applied with Procedure 1.

Figure 3 shows the temporal evolution of the grain shape during shrinkage of a circular grain with an initial size of $\mathrm{R}_{0}=50$ imbedded in an infinite matrix as simulated using Procedure 4. As is to be expected with lattice anisotropy, the grain maintains an approximately circular shape only within statistical fluctuations.

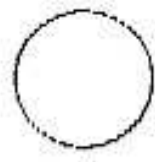

0

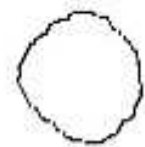

45

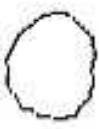

100

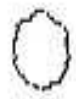

$150185 \mathrm{MCS}$

Fig. 3. Temporal evolution of circular grain $\left(R_{0}=50\right)$ in infinite matrix for Procedure 4

In Figure 4, the evolutions of grain area $\mathrm{A}$ and grain radius $\mathrm{R}$ are depicted as functions of time for the simulation as described above. As is expected from theory, grain area A was found to decrease linearly with time. A regression analysis was used to fit the grain radius $\mathrm{R}$ vs. time $t$, producing the following result:

$$
R=3.5109(189.4420-t)^{0.5097}
$$

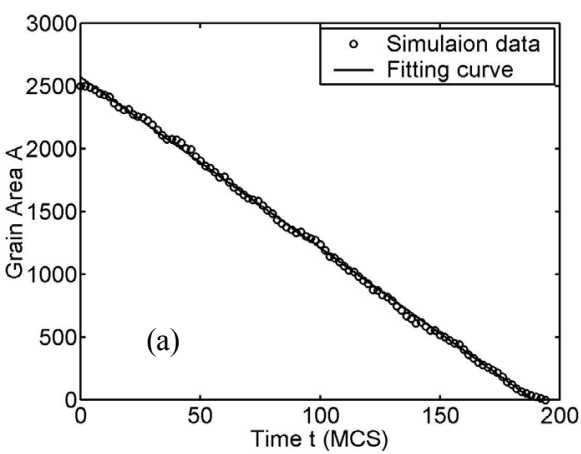

(a)

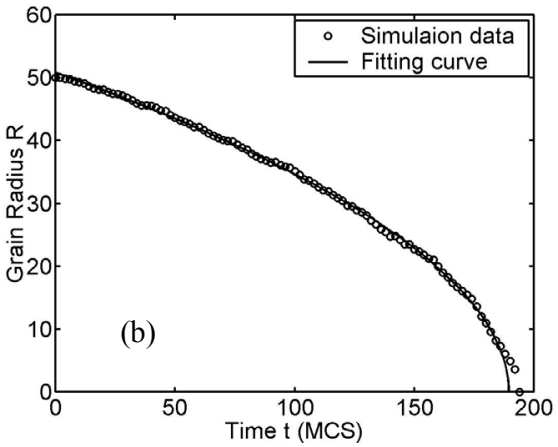

(b)

Fig. 4. Temporal evolutions of (a) grain area and (b) grain radius of circular grain $\left(\mathrm{R}_{0}=50\right)$ for Procedure 4

A comparison of the grain area evolutions simulated using Procedures 1 (with $Q=2$ ), 2, 3 and 4 is presented in Figure 5. In all four cases, an approximately linear relationship between grain area A and time $t$ was obtained. Also, regression analyses on data for grain radius $R$ vs. time $t$ yielded a grain shrinkage exponent of around 0.5 as well, which confirms 
corresponding findings reported elsewhere (Anderson et al., 1984; Radhakrishnan \& Zacharia, 1995). This result implies that the essential feature of curvature-driven grain growth is adequately captured by the MC.

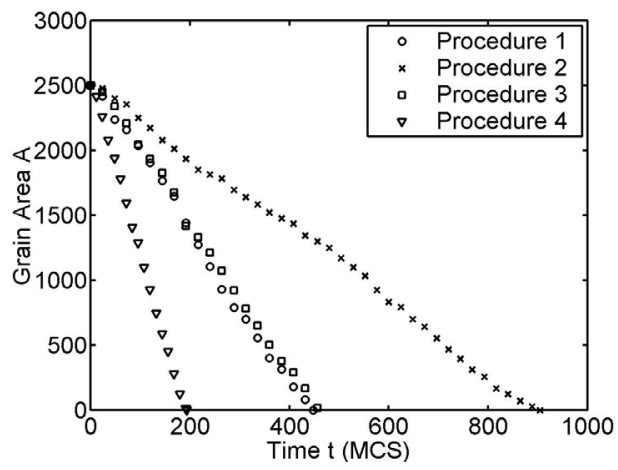

Fig. 5. Temporal evolution of circular grain area A for Procedures 1 through 4

It should be pointed out that Procedure 4 causes the grain to disappear in the shortest time. Furthermore, a comparison of the simulation results obtained with Procedures 3 and 4 reveals that the modified approach for selecting candidate lattice sites for reorientation (Modification III) reduced the number of MCS by roughly $50 \%$. Also, the area evolutions for Procedure 1 (with $Q=2$ ) and Procedure 3 are identical within the expected margins of error because these two procedures are essentially equivalent. Finally, a 50\% reduction in MCS compared with Procedure 2 is achieved by employing Modification II for selecting candidate new orientations.

\subsubsection{Kinetics of normal grain growth}

Next, the computational efficiency of simulating isotropic normal grain growth using Procedure 4 was studied ( $\mathrm{Yu}$ \& Esche, 2003a). Figure 6 depicts the microstructures simulated using the modifications discussed above at two instances. It was confirmed by careful examination of the microstructures obtained in the simulations that small grains tend to shrink and ultimately disappear while large grains grow, which is in accordance with simulation results reported previously (Srolovitz et al., 1984a). The morphology of the obtained grain structure is compact and similar to the real material microstructure. Some unrealistic straight grain boundaries are observable in Figure $6 \mathrm{~b}$ though, which are attributable to the anisotropy of the lattice used (Holm et al., 1991). Such straight boundaries may be eliminated by either artificially introducing noise into the system in the form of a non-zero temperature probability or by including the second nearest neighbors into the energy calculations. However, the simulation temperature is an artificial parameter, and thus, its inclusion is not desirable from the perspective of object-oriented algorithm design. Also, while including the second nearest neighbors into the energy calculations appears physically justifiable, the relative weights assigned to the contributions of first and second nearest neighbors may again be considered as artificial parameters that are objectionable from the point of view of object-oriented algorithm design. Further developments on the construction of lattices with less pronounced anisotropy would therefore be desirable (Yang et al., 1995). 

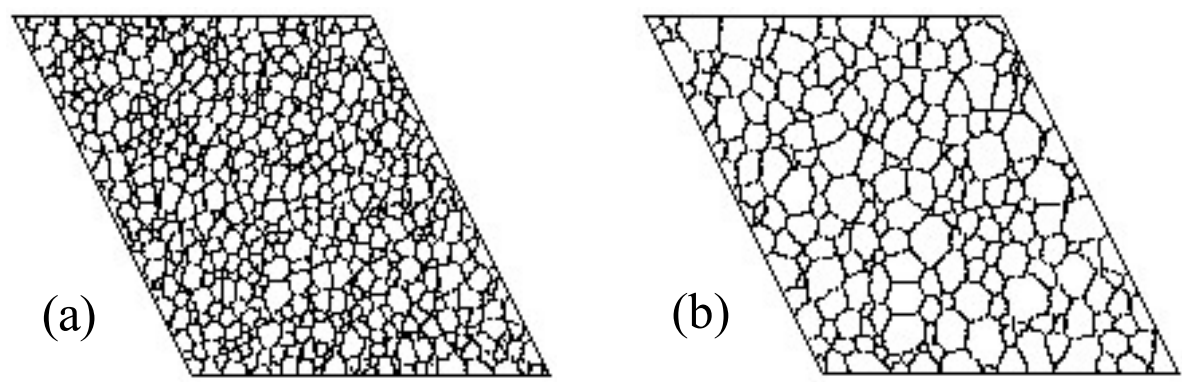

Fig. 6. Isotropic normal grain growth by Procedure 4 after (a) 100 MCS, (b) 285 MCS

Typical results for temporal evolutions of the mean grain radius obtained using the four procedures are shown in Figure 7. The grain growth exponents were calculated from Eq. 2 as the slopes of the log-log plots for grain radius vs. time, which is common practice (Anderson et al., 1984; Holm, 1992; Radhakrishnan \& Zacharia, 1995; Song et al., 1998). The data points for $<\mathrm{R}><7$ were excluded from the calculations of the grain growth exponents because they deviate from the linear relationship between $\log (<\mathrm{R}>)$ and $\log (\mathrm{t})$. This deviation may be attributed to the effect of the disorderly lattice structure early in the simulation and the neglecting of the initial grain radius $R_{0}$ in Eq. 2 . The corresponding grain growth exponents for the four procedures (averaged over 10 runs) were reported elsewhere (Yu \& Esche, 2003a).

The grain growth exponents were found to be $n=0.5$ in the large grain size regimes for all four procedures, which is in accordance with earlier reports (Grest et al., 1988; Radhakrishnan \& Zacharia, 1995). However, only the simulations using Procedure 4 generated $n=0.5$ in the earlier stage $(<R><15)$, and the simulations using Procedure 3 resulted in values of $\mathrm{n}$ between 0.44 and 0.49 . Therefore, both Modifications II and III affect the grain growth kinetics.

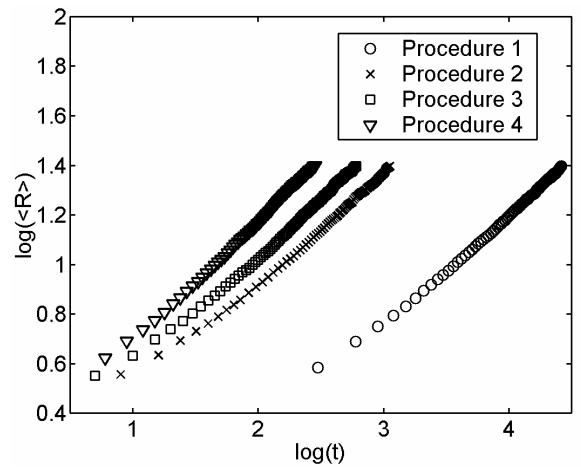

Fig. 7. Grain growth kinetics for all four procedures

\subsubsection{Grain growth exponent}

Since a grain growth exponent of $\mathrm{n}=0.5$ was obtained in the large grain size regimes using the conventional MC algorithm with the n-fold method (Grest et al., 1988), it was then hypothesized that unrealistic (unphysical) finite size effects are likely to dominate in the 
early simulation stages, which would in turn lead to lower values of $\mathrm{n}$ in the small grain size regimes. However, the modified MC algorithm discussed above generates $n=0.5$ even in the early simulation stages. The obtained growth exponent was further validated by fitting the data for average grain radius $<R>$ vs. time $t$ to Eq. 1 . A typical result is given in Figure 8 .

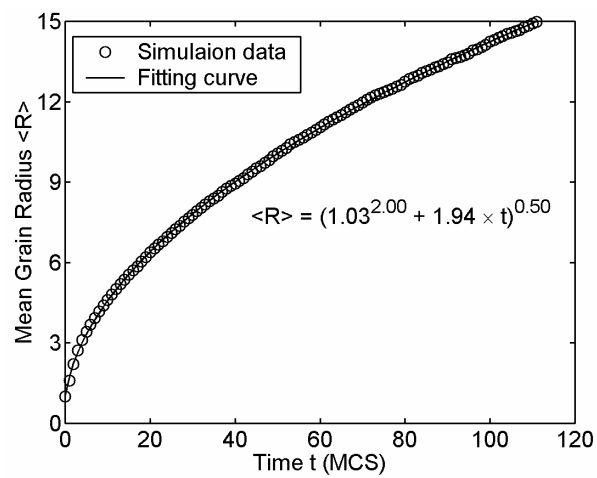

Fig. 8. Regression analysis for data using Procedure 4 with objective function $<\mathrm{R}>\mathrm{m}$ $<\mathrm{R}\rangle_{0} \mathrm{~m}=$ Mt with parameters $\mathrm{m},\langle\mathrm{R}\rangle_{0}$ and $\mathrm{M}$ to be determined

By analyzing the distributions of the normalized grain sizes and the numbers of edges per grain, the grain evolution was found to be a self-similar process (see Figure 9). From Figure $9 b$, it is evident that the grain shapes remain essentially unchanged, with the exception of the very beginning of the simulation $(t<1$ MCS). This means that, when modeled using Procedure 4, the grain boundary movement immediately results in a steady state, despite the fact that the initial lattice structure is random. Additional support for this observation is provided by the value of $\langle\mathrm{R}\rangle_{0}=1.03$ obtained by regression analysis (see Figure 8). Therefore, it appears that Procedure 4 leads to the expected parabolic growth law, independently of any size effects. This conclusion is further supported by a value of $n=0.5$ obtained in the Ising model using the conventional MC algorithm (Anderson et al., 1984; Grest et al., 1988). Note that in this case the modeling of the boundary movement is equivalent to Modification II discussed above. Furthermore, there is no theoretical basis for a dependence of the grain growth exponent on the grain size. In fact, in Mullins' theoretical deduction of the parabolic growth law under the assumption of statistical self-similarity (Mullins, 1986), the grain size was not taken into consideration as long as there are enough domains in the system in order to avoid surface effects and if the material volume is differentiable, i.e., the material is continuous at the length scale of the grain size considered. Even though the lattice in the MC method represents a set of discrete points, it is the continuous grain boundaries whose movements are modeled in the MC algorithm. Thus, a continuous material can be mapped onto the lattice without loss of the continuum property. Therefore, the MC lattice is appropriate for representing the theoretical material model employed by Mullins, and the MC algorithm can be expected to reproduce the theoretical result. Note that it indeed does, as is documented through the results discussed above.

The reasoning above demonstrates that the lower grain growth exponents reported for the conventional MC algorithm cannot be explained by finite size effects. The temporal evolutions of the normalized grain size and grain shape distributions obtained using the conventional MC algorithm are self-similar (Srolovitz et al., 1984a) and resemble those shown in Figure 9. A 
careful study of the growth kinetics obtained using the conventional MC algorithm suggested that its strong random nature significantly affects the growth kinetics and the results of the regression analysis used to fit the simulation data (Yu \& Esche, 2003b). By excluding the data from the very early stages, where the randomness effects are likely to dominate the simulated lattice evolution, and by employing three-parameter regression analyses based on Eq.1, growth exponents close to $\mathrm{n}=0.5$ can be obtained.

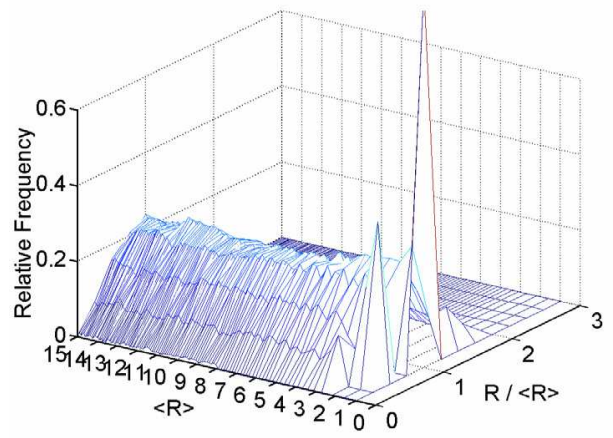

(a)

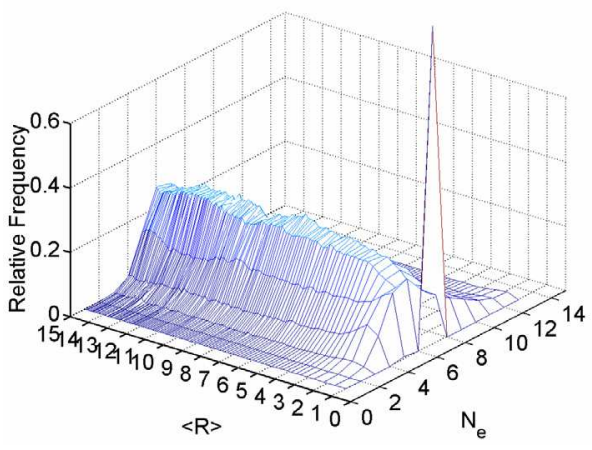

(b)

Fig. 9. Distributions for Procedure 4 of (a) grain radius $\mathrm{R}$ normalized by mean grain radius $<\mathrm{R}>$ and (b) number of edges per grain $\mathrm{N}_{\mathrm{e}}$

While the lower grain growth exponent measured experimentally for zone-refined materials was attributed to the initial grain morphology and grain size distribution among other reasons (Humphreys \& Hatherly, 1996), the parabolic grain growth kinetics still occurs even for unstructured initial lattices as is shown in Figure 9. Therefore, the MC simulations do not support this reasoning.

\subsubsection{Algorithm efficiency}

Both the total number of MCS and the total computing time to reach a certain grain size could be considered as parameters that characterize the computational efficiency of the MC algorithm. An investigation of the computational efficiency of the four alternative procedures discussed above was conducted using a $400 \times 400$ triangular lattice (Yu \& Esche, 2003a). While the average numbers of MCS per second were found to be similar for all four procedures, the total numbers of MCS and the total computing times for Procedure 4 were significantly lower than those for the remaining three procedures. Thus, according to both measures, Procedure 4 exhibits by far the best efficiency.

\section{Three-dimensional Monte Carlo method for grain growth simulations}

\subsection{Background}

The two-dimensional grain growth of ideal single-phase materials has been studied extensively both by theoretical deductions (Burke \& Turnbull, 1952) and by computer simulations (Anderson et al., 1984; Holm \& Battaile, 2001; Yu \& Esche, 2003a). However, the grain growth process represents a three-dimensional (3D) phenomenon. While the generalization of von Neumann's law from 2D to 3D has been attempted (Rivier, 1983; Liu 
et al., 2001), these 3D models are still not widely accepted and necessitate further development (Mullins, 1986; Atkinson, 1988). In light of the technical difficulties associated with experimental studies of 3D grain growth, computer simulation represents an effective tool for exploring this 3D microstructural event. A number of Monte Carlo (MC) Potts models for the 3D grain growth process have been developed (Anderson et al., 1989; Song \& Liu, 1998; Sista \& DebRoy, 2001). In these 3D simulations, the grain growth exponent $\mathrm{n}$ was found to either asymptotically approach the theoretical value in the long-time limit (Radhakrishnan \& Zacharia, 1995) or to be significantly lower than the theoretically expected value over the entire time domain (Yang et al., 2000). Furthermore, the normalized grain size distributions obtained in 3D MC Potts simulations was time-dependent (Xiaoyan et al., 2000). In addition, the 3D lattices employed in these earlier 3D simulations were usually limited to a size of $100 \times 100 \times 100$. Therefore, these simulations had to be terminated at relatively small grain sizes in order to preserve adequate numbers of grains for statistically significant analysis and could not adequately support the theoretical work.

A modified MC Potts algorithm that had been shown to produce the theoretically expected grain growth exponent over the entire time domain in 2D MC Potts simulations (Yu \& Esche, 2003a; Yu \& Esche, 2003b) was later expanded to model 3D grain growth (Yu \& Esche, 2003c; Yu et al., 2005) and will be briefly described here.

\subsection{Implementation}

In the 3D MC Potts algorithm for isotropic, single-phase grain growth simulation, a continuum microstructure was mapped onto a $200 \times 200 \times 200$ cubic lattice with 26 nearest neighbors (i.e. including third nearest neighbors). A MCS was defined as $\mathrm{N}$ reorientation attempts, where $\mathrm{N}=8,000,000$ here. The radius $\mathrm{R}$ of a 3D grain was defined as the cubic root of the grain's volume (i.e. the total number of lattice points within the grain). The average grain volume was defined as the total number of lattice points (i.e., 8,000,000) divided by the total number of $3 \mathrm{D}$ grains in the lattice, and the average $3 \mathrm{D}$ grain radius $<\mathrm{R}>$ was defined as the cubic root of the average grain volume. The radius of a grain in a cross section was defined as the square root of the number of lattice points within the grain in the cross section, and the average grain radius for a cross section was defined as the square root of the total number of lattice points in the cross section (i.e., 40,000) divided by the total number of grains in the cross section.

The cubic lattice was initialized by randomly assigning an integer orientation number to each lattice point. Initially, the number of different orientations was $Q=N=8,000,000$. In the reorientation procedure of the modified MC Potts algorithm, all lattice sites were randomly attempted for reorientation exactly once in each MCS. In the modified algorithm, the location of the lattice sites were checked first, and only the sites located at the grain boundaries were selected for a possible reorientation. For each attempted lattice site, the new orientation was selected randomly from those of its neighbors with unlike orientations. If the system energy did not rise due to the attempted reorientation, the new orientation number was accepted. Otherwise, the old orientation number was recovered. The simulations were terminated at time $\mathrm{t}=250 \mathrm{MCS}$. At that point, a grain size of $\langle\mathrm{R}>\approx 15$ was achieved and a large number of grains were preserved in the lattice. Each simulation was repeated ten times using different seeds for the random number generation. The microstructures were examined every 5 MCS. 


\subsection{Simulation results}

In order to facilitate the presentation of the simulation results, a Cartesian coordinate system was attached to the cubic lattice. For instance, $x=200, y=200$ and $z=200$ characterized the front, right and top surfaces of the cubic lattice, respectively, while $z=100$ represented the middle cross sectional plane in z-direction. Figure 10 depicts the 3D microstructure observed at the time instant when $\langle\mathrm{R}\rangle \approx 15$. Figure 11 shows the temporal evolution of the microstructure in the plane with $\mathrm{z}=100$. These figures indicate that a compact grain structure was developed. Also, various microstructural features of normal grain growth commonly found in 2D MC Potts simulations were observed in the cross sections of the 3D lattice. For instance, generally small grains shrank while large grains grew up, and 120degree angles were found at most grain corners. These observations are in accordance with findings reported earlier (Anderson et al., 1989, Song \& Liu, 1998).

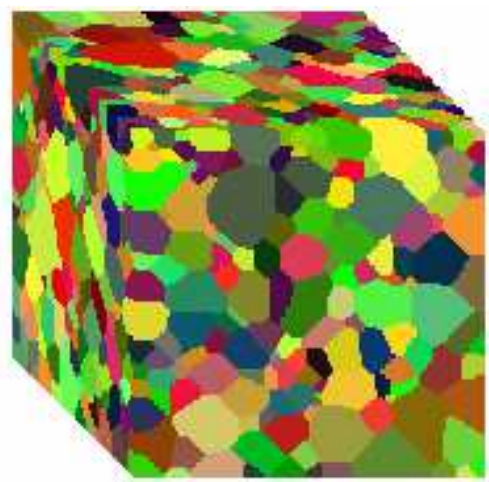

Fig. 10. 3D microstructure at $250 \mathrm{MCS},<\mathrm{R}>\approx 15$

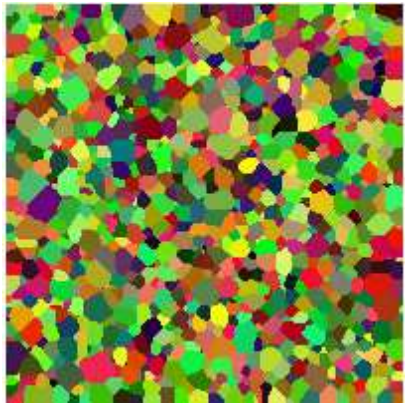

(a)

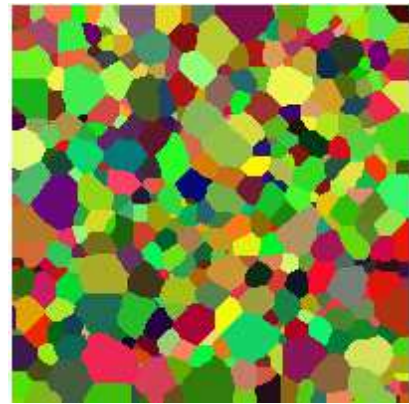

(b)

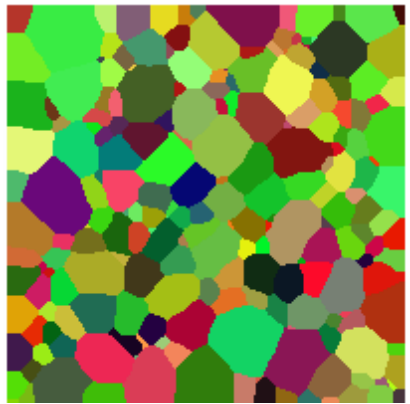

(c)

Fig. 11. Temporal evolution of microstructure in plane $\mathrm{z}=100$, (a) $50 \mathrm{MCS}\left(<\mathrm{R}>_{\mathrm{z}=100} \approx 6.4\right)$, (b) $150 \mathrm{MCS}\left(<\mathrm{R}>_{\mathrm{z}=100} \approx 10.1\right)$ and (c) $250 \mathrm{MCS}\left(<\mathrm{R}>_{\mathrm{z}=100} \approx 13.7\right)$

The temporal evolution of the distribution of $3 \mathrm{D}$ grain sizes $\mathrm{R}$ normalized by average grain radius $\langle\mathrm{R}\rangle$ is illustrated in Figure 12a. It can be observed that the grain size distribution did not change while $<\mathrm{R}>$ increased from 4 to 15 . Also, the grain size distributions obtained in ten simulation runs were very similar, i.e., the seeds used in the random number generation influenced the grain size distribution only very insignificantly. Two alternative equations - 
the Louat distribution in the form of Eq. 19 and the log-normal distribution in the form of Eq. 20 - were then fitted to the averaged grain size distribution data for the ten runs

$$
\begin{gathered}
F=C_{1} R_{n} \exp \left(-C_{2} R_{n}{ }^{2}\right) \\
F=C_{3} \exp \left[-C_{4}\left(\ln R_{n}-C_{5}\right)^{2}\right]-C_{6}
\end{gathered}
$$

where $\mathrm{F}$ is the relative frequency, $\mathrm{R}_{\mathrm{n}}=\mathrm{R} /<\mathrm{R}>$ is the normalized grain size, and $\mathrm{C}_{1}-\mathrm{C}_{6}$ are the fitting parameters. Figure $12 \mathrm{~b}$ summarizes the results of the regression analyses. It is apparent that the log-normal distribution provided for a better fit for the 3D simulation data than the Louat distribution. This conclusion confirms prior observations reported elsewhere (Radhakrishnan \& Zacharia, 1995). Specifically, the simulations resulted in more small grains and fewer large grains than in the log-normal curve. This deviation indicates that the grains in the simulations had a higher potential for later growth - a phenomenon that had also been documented before in the literature (Srolovitz et al., 1984a).

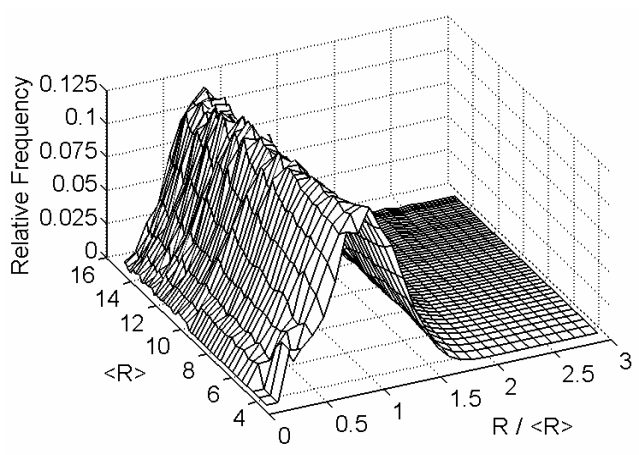

(a)

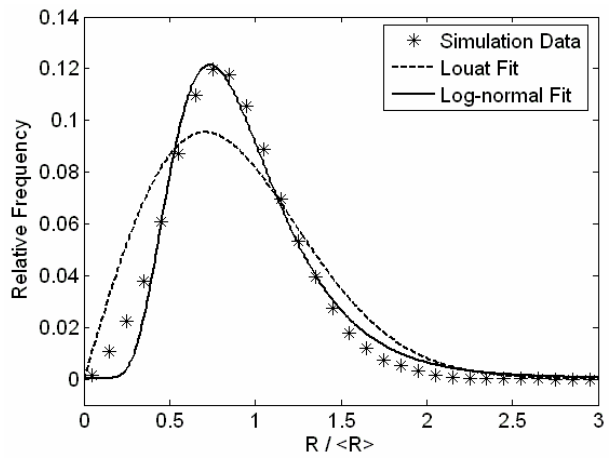

(b)

Fig. 12. (a) Time-invariant distribution of 3D grain sizes $\mathrm{R}$ normalized by average grain sizes $<\mathrm{R}>$; (b) regression analyses of grain size distribution averaged over time and over 10 simulation runs

Time-invariant grain size distributions were also observed for the cross sections. A typical example is given in Figure 13a. While the grain size distributions for the individual cross sections obtained in the ten simulations exhibited relatively large variations, the averaged grain size distributions over the ten runs for the three cross sections with $x, y, z=100$, respectively, differed only slightly. Regression analyses of the grain size averaged over the three cross sections showed that the simulation data can be fitted to both the Louat and the log-normal distributions (see Figure 13b). Contrary to the corresponding 3D case, the Louat distribution appears to provide a better fit, though. The fact that the Louat distribution provides a better fit for $2 \mathrm{D}$ simulation results had also been observed before (Anderson et al., 1989). Note also that alternative grain size distributions were discussed elsewhere (Mullins, 1991; Mullins, 1998). 


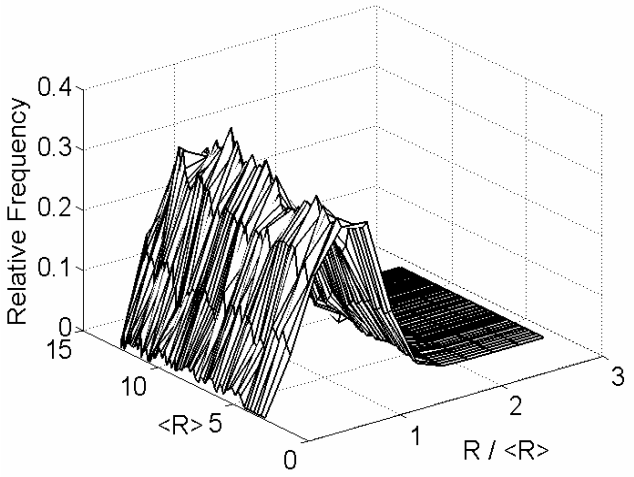

(a)

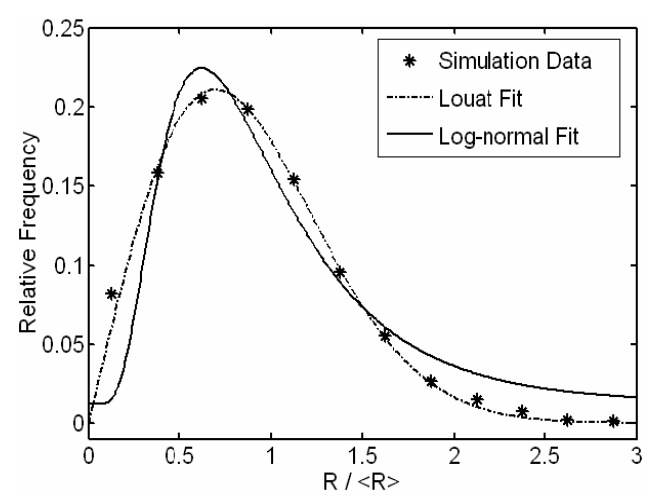

(b)

Fig. 13. Normalized grain size distributions for cross sections: (a) temporal evolution of grain size distribution in one cross section; (b) regression analyses of grain size distribution averaged over time, 10 simulation runs and 3 cross sections

Figure 14 shows the distributions of the number of facets per grain $\mathrm{N}_{\mathrm{f}}$ and the number of edges per grain $\mathrm{N}_{\mathrm{e}}$ for the cross sectional plane with $\mathrm{z}=100$. The $\mathrm{N}_{\mathrm{f}}$ distribution depicted in Figure 14a appears to be statistically time-invariant, though, except for the very late simulation stage that exhibits a sharp peak. The peak values of $\mathrm{N}_{\mathrm{f}}$ fall in the relatively narrow range between 11 and 14 as seen in Figure 15, which compares with a value of $\mathrm{N}_{\mathrm{f}}=13.7$ for the average number of facets per grain reported elsewhere (Krill \& Chen, 2002). Furthermore, a range of 11.16 to 15.54 was reported for experimental measurements of $\mathrm{N}_{\mathrm{f}}$ (Krill \& Chen, 2002). Similarly, the distribution of edges per grain $\mathrm{N}_{\mathrm{e}}$ is also statistically time-invariant as is seen in Figure $14 \mathrm{~b}$.

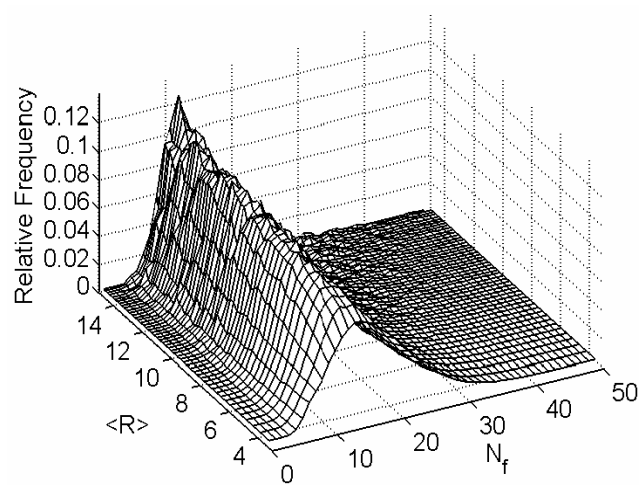

(a)

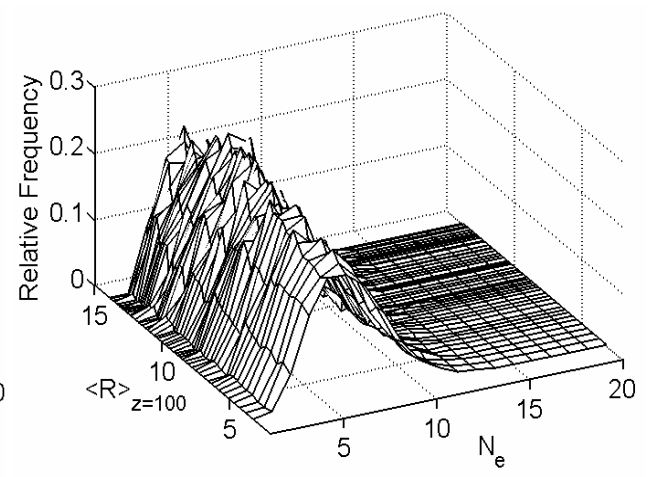

(b)

Fig. 14. Distribution of number of (a) facets per grain $\mathrm{N}_{\mathrm{f}}$ for $3 \mathrm{D}$ domain and (b) edges per grain $\mathrm{N}_{\mathrm{e}}$ for cross sectional plane with $\mathrm{z}=100$ 


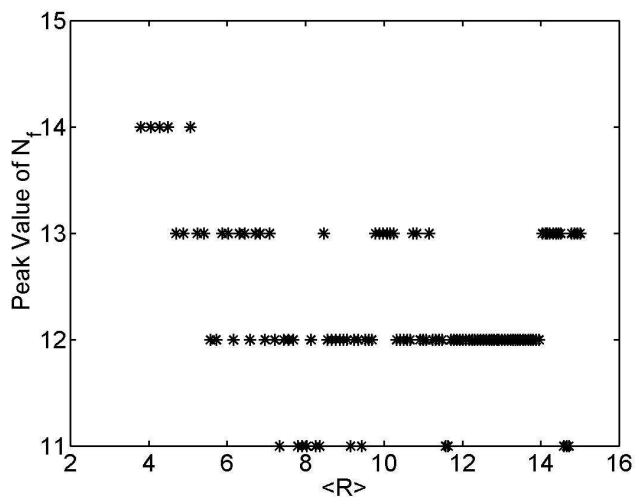

Fig. 15. Evolution of peak value of $\mathrm{N}_{\mathrm{f}}$ (average value 12.25)

Least-square regression analyses of the grain growth kinetics were then performed based on the data obtained from the 3D domain and the three cross sections at $x=100, y=100$ and $z=100$. In each case, the data for grain size vs. time were averaged over ten simulation runs prior to conducting the regression analysis. Note that the differences in grain size vs. time data between the ten simulation runs were very small. Figure 16 illustrates the data for averaged grain size vs. time and regression analysis results based on the classical form of the grain growth kinetics (i.e. Eq. 1 with fitting parameters $\left\langle\mathrm{R}>_{0}, \mathrm{~m}\right.$ and $\mathrm{M}$ and defining the grain growth exponent as $n=1 / m)$. A value of $n \approx 0.5$ was obtained both for the $3 D$ domain as well as for the cross sections. The grain growth rates $M$ obtained from the three cross sections were similar to each other with an average value of $\mathrm{M} \approx 0.74$. Also, they were smaller than the rate of $M \approx 0.96$ found for the 3D domain (see Figure 16b). In Figure 17, a $\log -\log$ plot of average grain radius $<R>$ vs. time $t$ is provided. Note that the same grain growth kinetics was also observed in simulations with grain sizes larger than 15 .

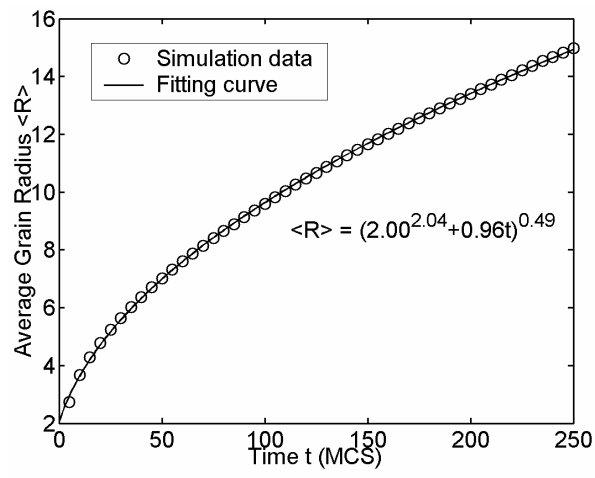

(a)

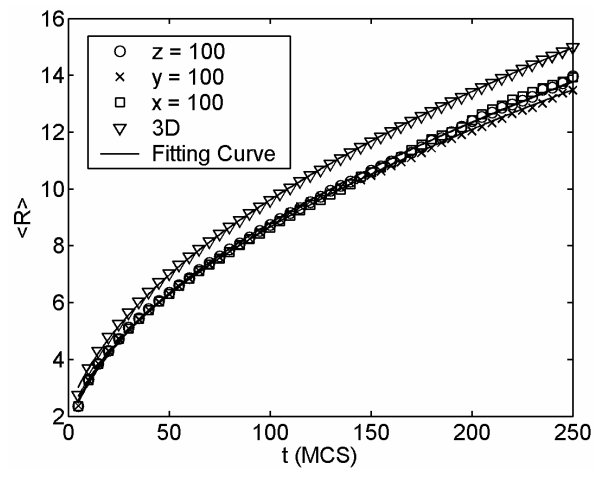

(b)

Fig. 16. Grain growth kinetics obtained with $\mathrm{n} \approx 0.5$ (a) for $3 \mathrm{D}$ domain and (b) for cross sections with $x=100, y=100$ and $z=100$, respectively 


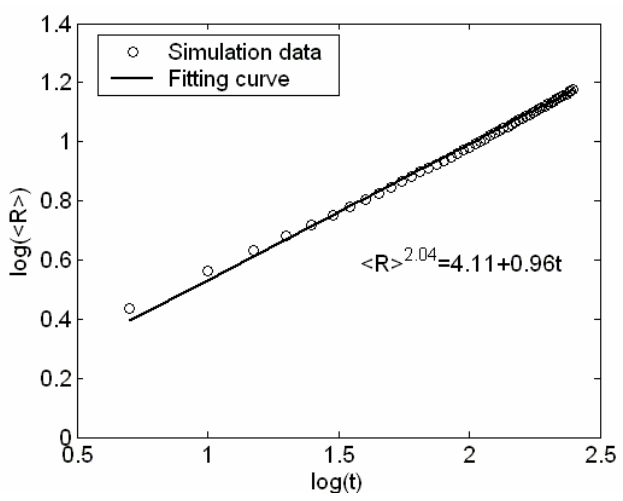

Fig. 17. Log-log plot of average grain radius $<\mathrm{R}>\mathrm{vs}$. time $\mathrm{t}$ for $3 \mathrm{D}$ domain

\section{Integration of Monte Carlo method into multi-scale approaches}

Thermo-mechanical processing is one of the near-net-shape metal-processing technologies where a combination of mechanical pressure and heat is applied concurrently such as to deform a metallic workpiece into a desired shape. Since the microstructure of the manufactured products determines their mechanical properties, the various thermomechanical procedures (e.g., forging, rolling, extrusion, etc.) are heavily optimized in order to achieve advantageous microstructures. In the past, trial-and-error methods were used in industrial practice for designing and optimizing these thermo-mechanical processes, which led to high manufacturing costs and long lead times. Neither one of those is tolerable anymore in today's highly competitive and truly global economy. With modern computer technologies, numerical process modeling that is capable of predicting fairly accurately the shape of the deformed parts as well as the strain, strain rate, stress and temperature distributions has become feasible and more prevalent. This success of numerical process modeling is largely attributable to the achieved quality of the applied constitutive laws (i.e., coupling of mechanical and thermal models) that describe the basic material flow under the influence of pressure and heat and to the development of efficient and accurate numerical techniques such as the Finite Element Method (FEM; Altan \& Vazquez, 1997; Walters et al., 1997).

Recent efforts toward improving the accuracy of the numerical predictions are now increasingly focusing on the modeling of the microstructural phenomena that occur during the thermo-mechanical processing of metals as consequences of complex metallurgical events such as recovery, recrystallization, grain growth, phase transformations, precipitation and dissolution reactions, etc. These microstructural phenomena may occur dynamically during deformation processing or either meta-dynamically or statically during post-deformation cooling or heat treatment. The mechanisms and kinetics of these phenomena and the associated changes in size, morphology, distribution, volume fraction and composition of the constituent phases are strongly dictated by the macroscopic heat flow and material flow processes (Humphreys \& Hatherly, 1995; Doherty et al., 1997). Due to the variety and complexity of these microstructural events, such modeling presents significant challenges. Despite their scientific appeal, atomic-level modeling techniques require prohibitively high levels of computational power, and thus, they may remain 
infeasible at least for the near future. Therefore, two types of modeling approaches are commonly applied, namely microstructure vs. processing-parameter relationships that were obtained empirically through regression analyses of experimental data as well as mesoscopic models that employ the physical laws governing the microstructure evolution.

In empirical models for the temporal evolution of the microstructure of metals during thermo-mechanical processing, the relationships between microstructural features (such as grain size, texture, topology, morphology at the grain or subgrain level, dislocation density, misorientation at the substructure level) and processing parameters (such as tool and workpiece geometry, temperature, deformation rate, amount of deformation, interface friction, heat transfer conditions) are derived via regression analyses of experimental data (Grong \& Shercliff, 2002).

Currently, this empirical methodology is widely applied in industrial practice, albeit with moderate success. However, the range of applicability and the accuracy of such predictions are rather limited due to the empirical nature of the microstructure models employed. It should be noted that the underlying physical mechanisms of the microstructure evolution is not disclosed by these empirical models. Therefore, the applicability of these empirical models is confined to within the boundaries where they were obtained, and hence, they do not offer any universal prediction capabilities. Furthermore, because they are usually of a simple form, they are generally not suitable for describing more complicated microstructural phenomena.

Due to these shortcomings of empirical models, microstructural modeling approaches at the mesoscopic scale such as the Cellular Automaton (Feppon \& Hutchinson, 2002) and the Monte Carlo Potts Model have been developed. In these approaches, the continuous material structure is discretized into a lattice that typically comprises thousands of grains. Physical laws (such as the surface energy reduction law governing normal grain growth, the site-saturated nucleation law for recrystallization, etc.) are then invoked to model the temporal evolution of the lattice. While mesoscopic models have been applied successfully to various microstructural phenomena in thermal processing such as welding (Yang et al., 2000) and film growth (Mizuseki et al., 2002), they have not yet been employed to predict the microstructure for industrially relevant thermo-mechanical processes such as hot forging or hot extrusion.

As a result of the recent rapid progress in the development of the mesoscopic microstructure modeling approach, various researchers have proposed to model the microstructure evolution in thermo-mechanical processing by combining a mesoscopic plasticity Finite Element (FE) analysis (Sarma et al., 1998) and the MC method (Radhakrishnan et al., 1998) or other microstructural models (Raabe \& Becker, 2000) at the mesoscopic level. From a theoretical standpoint, these efforts are very appealing and the corresponding prediction methodology (Miodownik et al., 1999; Beaudoin et al., 2002) is expected to be extendable to problems of practically relevant size when macroscopic plasticity FE models have been obtained. However, macroscopic plasticity FE models may still not be available in the foreseeable future. Therefore, a systematic methodology for microstructure prediction in thermo-mechanical processing based on models at multiple length scales is briefly summarized below (Yu \& Esche, 2005).

In such a multi-scale simulation methodology, the simulation input includes the processing conditions, the macroscopic and mesoscopic material properties as well as the initial microstructure features. These input parameters are supplied to the various modules of the modeling system, which consists of continuum-based coupled thermo-mechanical models, a 
multi-scale modeling interface and mesoscopic microstructure models. The final output of the simulation sequence consists of the resulting microstructure features. For instance, consider the modeling of the microstructure evolution during hot forging of a single-phase material followed by an annealing procedure. In a simulation system consisting of FEM and MC modules, the simulation input includes the processing conditions (tool and workpiece geometry, temperature, deformation speed, amount of deformation, interface friction, etc.), the macroscopic material parameters (e.g., Young's modulus, Poisson's ratio, yield and hardening characteristics, thermal properties, etc.), initial microstructure features (i.e., initial grain size) and mesoscopic material properties (i.e., grain boundary energy and mobility). The simulation system is composed of an FEM module for modeling the macroscopic mechanical deformation process, a module for computing the stored energy from the primary field variables (i.e., strain and stress) and an MC-based module for modeling the static recrystallization and grain growth during the annealing process. The FEM-based calculations of the deformed system configuration and of the stored energy can be performed for the entire workpiece, which results in complete distributions of all field variables. Then, in order to save computational resources, the MC-based recrystallization and grain growth simulations can be limited to only a few representative elements of the FEM mesh as shown in Figure 18. Obtaining simulation results over the entire domain of the workpiece would probably necessitate a massively parallel computing system and might not even be necessary for most industrial applications. A more detailed discussion of the three simulation modules involved in the multi-scale modeling approach (i.e., FEM, multiscale modeling interface and MC-based microstructure models) was provided elsewhere (Yu \& Esche, 2005).

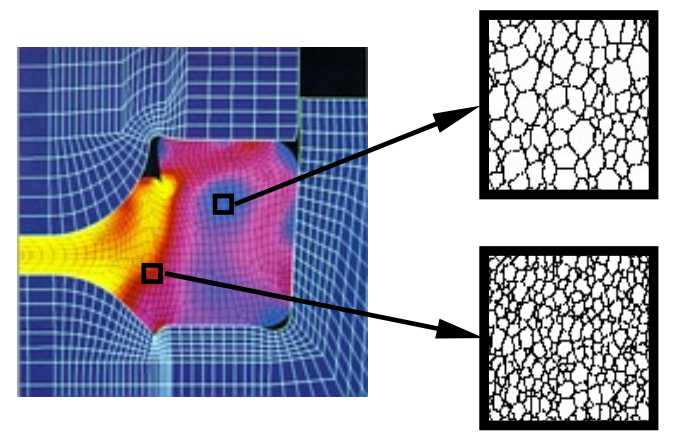

Fig. 18. Selection of representative zones for microstructure modeling1

The systematic multi-scale microstructure prediction methodology briefly described above represents one critically needed building block of a more comprehensive multi-scale modeling approach for material processing and material properties, which would be readily applicable to the industrial product design process. Such a comprehensive design system would comprise a continuum-based process model, a microstructure model and a material property model. Macro-scale process models are based on classical continuum mechanics. They predict the field variables as functions of the processing conditions and continuum material properties. These field variables in turn serve as the input to mesoscopic (or

${ }^{1}$ Figure adapted from website of Scientific Forming Technologies Corporation. 
empirical) microstructure models for simulating the change in the microstructure features due to the material processing. Then, the resulting microstructure and field variables can be fed into material property models at either atomic or mesoscopic scales (or alternatively to empirical material property relationships). Finally, the material properties resulting from the material property models can either be compared with the given product requirements in order to optimize the processing conditions, or they can be inserted back into the process model such as to incorporate instantaneous influences of microstructural changes on the material properties used in the process model. In addition, the material property model can also provide mesoscopic material properties for the microstructure model.

\section{Conclusion}

In this chapter, the application of the MC method to two-dimensional simulations of the normal grain growth of isotropic single-phase materials was presented. After briefly reviewing some theoretical foundations on the self-similarity and the kinetics of grain growth, the conventional MC method was introduced. This was followed by a discussion of several modifications of the conventional MC algorithm that have been demonstrated to improve its accuracy and efficiency. Subsequently, the extension of the MC method to threedimensional problems was described and its characteristics regarding grain growth kinetics and grain size distribution were analyzed. Finally, an approach for integrating the MC method into simulations of thermo-mechanical processes at multiple length scales was outlined.

\section{References}

Altan, T. \& Vazquez, V. (1997). Status of process simulation using 2D and 3D finite element method 'What is practical today? What can we expect in the future?'. Journal of Materials Processing Technology, Vol. 71, No. 1, pp. 49-63.

Anderson, M. P., Srolovitz, D. J., Grest, G. S. \& Sahni, P. S. (1984). Computer simulation of grain growth I - Kinetics. Acta Metallurgica, Vol. 32, No. 5, pp. 783-791.

Anderson, M. P., Grest, G. S. \& Srolovitz, D. J. (1989). Computer simulation of grain growth in three dimensions. Philosophical Magazine, Vol. B59, No. 3, pp. 293-329.

Atkinson, H. V. (1988). Theories of normal grain growth in pure single phase systems. Acta Metallurgica, Vol. 36, No. 3, pp. 469-491.

Beaudoin, A. J., Srinivasan, R. \& Semiatin, S. L. (2002). Microstructure modeling and prediction during thermomechanical processing. JOM, Vol. 54, No. 1, pp. 25-29.

Beenakker, C. W. J. (1988). Numerical simulation of a coarsening two-dimensional network. Physical Review A, Vol. 37, No. 5, pp. 1697-1702.

Bolling, G. F. \& Winegard, W. G. (1958). Grain growth in zone-refined lead. Acta Metallurgica, Vol. 6, No. 4, pp. 283-287.

Bortz, A. B., Kalos, M. H. \& Lebowitz, J. L. (1975). A new algorithm for Monte Carlo simulation of Ising spin systems. Journal of Computational Physics, Vol. 17, No.1, pp. 10-18.

Burke, J. E. \& Turnbull, D. (1952). Recrystallization and grain growth. Progress in Metal Physics, Vol. 3, pp. 220-292. 
Chen, I.-W., Hassold, G. N. \& Srolovitz, D. J. (1990). Computer simulation of final stage sintering II: Influence of initial pore size. Journal of the American Ceramic Society Vol. 73, No. 10, pp. 2865-2872.

Doherty, R. D., Hughes, D. A., Humphreys, F. J., Jonas, J. J., Juul Jensen, D., Kassner, M. E., King, W. E., McNelley, T. R., McQueen, H. J. \& Rollett, A. D. (1997). Current issues in recrystallization: a review. Materials Science and Engineering A, Vol. 238, No. 2, pp. 219-274.

Feppon, J. M. \& Hutchinson, W. B. (2002). On the growth of grains. Acta Materialia, Vol. 50, No. 13, pp. 3293-3300.

Geiger, J., Roosz, A. \& Barkoczy, P. (2001). Simulation of grain coarsening in two dimensions by cellular-automaton. Acta Materialia, Vol. 49, No. 4, pp. 623-629.

Glazier, J. A., Gross, S. P. \& Stavans, J. (1987). Dynamics of two dimensional soap froths. Physical Review A, Vol. 36, pp. 306-312.

Grest, G. S., Srolovitz, D. J. \& Anderson, M. P. (1985). Computer simulation of grain growth - IV. Anisotropic grain boundary energies. Acta Metallurgica, Vol.33, No.3, pp. 509-520.

Grest, G. S., Anderson, M. P. \& Srolovitz, D. J. (1988). Domain-growth kinetics for the Qstate Potts model in two and three dimensions. Physical Review B, Vol. 38, No. 7, pp. 4752-4759.

Grong, O. \& Shercliff, H. R. (2002). Microstructural modeling in metals processing. Progress in Materials Science, Vol. 47, No. 2, pp. 163-282.

Hassold, G. N., Chen, I.-W. \& Srolovitz, D. J. (1990). Computer simulation of final-stage sintering I: Model, kinetics, and microstructure. Journal of the American Ceramic Society, Vol. 73, No. 10, pp. 2857-2864.

Hillert, M. (1965). On the theory of normal and abnormal grain growth. Acta Metallurgica, Vol. 13, No. 3, pp. 227-238.

Holm, E. A., Glazier, J. A., Srolovitz, D. J. \& Grest, G. S. (1991). Effects of lattice anisotropy and temperature on domain growth in the 2-dimensional Potts-model. Physical Review A, Vol. 43, No. 6, pp. 2662-2668.

Holm, E. A. (1992). Modeling microstructural evolution in single-phase, composite and twophase polycrystals. Ph. D. thesis, University of Michigan.

Holm, E. A., Srolovitz, D. J. \& Cahn, J. W. (1993). Microstructural evolution in twodimensional two-phase polycrystals. Acta Metallurgica and Materialia, Vol. 41, pp. 1119-1136.

Holm, E. A. \& Battaile, C. C. (2001). The computer simulation of microstructural evolution. JOM, Vol. 53, No. 9, pp. 20-23.

Holm, E. A., Hassold, G. N. \& Miodownik, M. A. (2001). On misorientation distribution evolution during anisotropic grain growth. Acta Materialia, Vol.49, No.15, pp. 2981-2991.

Humphreys, F. J. \& Hatherly, M. (1996). Recrystallization and Related Annealing Phenomena. Pergamon.

Ivasishin, O. M., Shevchenko, S. V. \& Semiatin, S. L. (2004). Modeling of abnormal grain growth in textured materials. Scripta Materialia, Vol. 50, No. 9, pp. 1241-1245.

Ivasishin, O. M., Shevchenko, S. V., Vasiliev, N. L. \& Semiatin, S. L. (2003). 3D Monte Carlo simulation of texture-controlled grain growth. Acta Materialia, Vol.51, No.4, pp. 1019-1034. 
Janssens, K. G. F., Raabe, D., Kozeschnik, E., Miodownik, M. A. \& Nestler, B. (2007). Computational Materials Engineering - An Introduction to Microstructure Evolution, Elsevier Academic Press, Oxford.

Kad, B. K. \& Hazzledine, P. M. (1997). Monte Carlo simulation of grain growth and Zener pinning. Material Science and Engineering A, Vol. 238, No. 1, pp. 70-77.

Krill III, C. E. \& Chen, L. Q. (2002). Computer simulation of 3-D grain growth using a phasefield model. Acta Materialia, Vol. 50, pp. 3057-3073.

Kumar, S., Gunton, J. D. \& Kaski, K. (1987). Dynamical scaling in the Q-state Potts model. Physical Review B, Vol. 35, pp. 8517-8522.

Lee, H. N., Ryoo, H. S. \& Hwang, S. K. (2000). Monte Carlo simulation of microstructure evolution based on grain boundary character distribution. Material Science and Engineering A, Vol. 281, No. 1, pp. 176-188.

Liu, G., Yu, H., Song, X. \& Qin, X. (2001). A new model of three-dimensional grain growth: theory and computer simulation of topology-dependency of individual grain growth rate. Materials \& Designs, Vol. 22, No. 1, pp. 33-38.

Louat, N. P. (1974). On the theory of normal grain growth. Acta Metallurgica, Vol. 22, No. 6, pp. 721-724.

Matsubara, H. (1999). Computer simulations for the design of microstructural developments in ceramics. Computational Materials Science, Vol. 14, No. 1, pp. 125-128.

Mehnert, K. \& Klimanek, P. (1996). Monte Carlo simulation of grain growth in textured metals. Scripta Materialia, Vol. 35, No. 6, pp. 699-704.

Mehnert, K. \& Klimanek, P. (1997). Grain growth in materials with strong textures: 3D Monte Carlo simulations. Computational Materials Science, Vol. 9, No. 1-2, pp. 261-266.

Messina, R., Soucail, M. \& Kubin, L. (2001). Monte Carlo simulation of abnormal grain growth in two dimensions. Material Science and Engineering A, Vol. 308, No. 1-2, pp. 258-267.

Miodownik, M. A. (2002). A review of microstructural computer models used to simulate grain growth and recrystallisation in aluminum alloys. Journal of Light Metals, Vol. 2, No. 3, pp. 125-135.

Miodownik, M. A., Holm, E. A., Godfrey, A. W., Hughes, D. A. \& LeSar, R. (1999). Multiscale modeling of recrystallization. MRS Symposium Proceedings, Vol.538, pp. 157-162.

Miodownik, M., Holm, E. A. \& Hassold, G. N. (2000). Highly parallel computer simulations of particle pinning: Zener vindicated. Scripta Materialia, Vol.42, No.12, pp. 11731177.

Mizuseki, H., Hongo, K., Kawazoe, Y. \& Wille, L. T. (2002). Multiscale simulation of cluster growth and deposition processes by hybrid model based on direct simulation Monte Carlo method. Computational Materials Science, Vol. 24, No. 1, pp. 88-92.

Mullins, W. W. (1956). Two-dimensional motion of idealized grain boundaries. Journal of Applied Physics, Vol. 27, No. 8, pp. 900-904.

Mullins, W. W. (1986). The statistical self-similarity hypothesis in grain growth and particle coarsening. Journal of Applied Physics, Vol. 59, No. 4, pp. 1341-1349.

Mullins, W. W. (1991). The statistical particle growth law in self-similar coarsening. Acta Metallurgica and Materialia, Vol. 39, No. 9, pp. 2081-2090.

Mullins, W. W. (1998). Grain growth of uniform boundaries with scaling. Acta Materialia, Vol. 46, No. 17, pp. 6219-6226. 
Mullins, W. W. \& Vinals, J. (1989). Self-similarity and growth kinetics driven by surface free energy reduction. Acta Metallurgica, Vol. 37, pp. 991-997.

Nosonovsky, M., Zhang, X. \& Esche, S. K. (2009). Scaling of Monte Carlo simulations of grain growth in metals. Modelling and Simulation in Materials Science and Engineering, Vol. 17, No. 2, 13 pp.

Peczak, P. (1995). A Monte Carlo study of influence of deformation temperature on dynamic recrystallization. Acta Metallurgica et Materialia, Vol. 43, No. 3, pp. 1279-1291.

Potts, R. B. (1952). Some generalized order-disorder transformations. Proceedings of the Cambridge Philosophical Society, Vol. 48, pp. 106-109.

Raabe, D. (2000). Scaling Monte Carlo kinetics of the Potts model using rate theory. Acta Materialia, Vol. 48, No. 7, pp. 1617-1628.

Raabe, D. \& Becker, R. C. (2000). Coupling of a crystal plasticity finite-element model with a probabilistic cellular automaton for simulating primary static recrystallization in aluminum. Modelling and Simulation in Materials Science and Engineering, Vol. 8, No. 4, pp. 445-462.

Raabe, D. (2002). Cellular Automata in materials science with particular reference to recrystallization simulation. Annual Review of Materials Research, Vol. 32, pp. 53-76.

Radhakrishnan, B. \& Zacharia, T. (1995). Simulation of curvature-driven grain growth by using a modified Monte Carlo algorithm. Metallurgical and Materials Transactions A, Vol. 26, pp. 167-180.

Radhakrishnan, B., Sarma, G. B. \& Zacharia, T. (1998). Modeling the kinetics and microstructural evolution during static recrystallization - Monte Carlo simulation of recrystallization. Acta Materialia, Vol. 46, No. 1, pp. 4415-4433.

Rhines, F. N., Craig, K. R. \& DeHoff, R. T. (1974). Mechanism of steady-state grain growth in aluminum. Metallurgical Transactions, Vol. 5, pp. 413-425.

Rivier, N. (1983). On the structure of random tissues or froths, and their evolution. Philosophical Magazine, Vol. 47, No. 5, pp. L45-L49.

Rollett, A. D., Srolovitz, D. J. \& Anderson, M. P. (1989). Simulation and theory of abnormal grain growth - Variable grain boundary energies and mobilities. Acta Metallurgica, Vol. 37, No. 4, pp. 2127-1240.

Rollett, A. D., Srolovitz, D. J., Anderson, M. P. \& Doherty, R. D. (1992a). Computer simulation of recrystallization - III. Influence of a dispersion of fine particles. Acta Metallurgica, Vol. 40, No. 12, pp. 3475-3495.

Rollett, A. D., Luton, M. J. \& Srolovitz, D. J. (1992b). Computer simulation of dynamic recrystallization. Acta Metallurgica et Materialia, Vol. 40, No. 1, pp. 43-55.

Rollett, A. D. \& Mullins, W. W. (1996). On the growth of abnormal grains. Scripta Metallurgica et Materialia, Vol. 36, No. 9, pp. 975-980.

Rollett, A. D. (1997). Overview of modeling and simulation of recrystallization. Progress in Materials Science, Vol. 42, No. 1-4, pg. 79-99.

Rollett, A. D. \& Raabe, D. (2001). A hybrid model for mesoscopic simulation of recrystallization. Computational Materials Science, Vol. 21, No. 1, pp. 69-78.

Safran, S. A., Sahni, P. S. \& Grest, G. S. (1983). Kinetics of ordering in two dimensions. I. Model systems. Physical Review B, Vol. 28, No. 5, pp. 2693-2704.

Sahni, P. S., Srolovitz, D. J., Grest, G. S., Anderson, M. P. \& Safran, S. A. (1983). Kinetics of ordering in two dimensions. II: Quenched systems. Physical Review B, Vol. 28, No. 5, pp. 2705-2716. 
Sarma, G. B., Radhakrishnan, B. \& Zacharia, T. (1998). Finite element simulations of cold deformation at the mesoscale. Computational Materials Science, Vol. 12, No. 2, pp. 105-123.

Sista, S. \& DebRoy, T. (2001). Three dimensional Monte Carlo simulation of grain growth in zone refined iron. Metallurgical and Materials Transactions B, Vol. 32, pp. 1195-1201.

Song, X. \& Liu, G. (1998). A simple and efficient three-dimensional Monte Carlo simulation of grain growth. Scripta Materialia, Vol. 38, No. 11, pp. 1691-1696.

Song, X. Y., Liu, G. Q. \& He, Y. Z. (1998). Modified Monte Carlo method for grain growth simulation. Progress in Natural Science, Vol. 8, pp. 92-97.

Song, X. \& Rettenmayr, M. (2002). Modelling study on recrystallization, recovery and their temperature dependence in inhomogeneously deformed materials. Materials Science and Engineering A, Vol. 332, No. 1-2, pp. 153-160.

Soucail, M., Messina, R., Cosnuau, A. \& Kubin, L. P. (1999). Monte Carlo simulation of Zener pinning in two dimensions. Material Science and Engineering A, Vol. 271, No. 1, pp. 1-7.

Srolovitz, D. J., Anderson, M. P., Sahni, P. S. \& Grest, G. S. (1984a). Computer simulation of grain-growth: II. Grain size distribution, topology, and local dynamics. Acta Metallurgica, Vol. 32, No. 5, pp. 793-802.

Srolovitz, D. J., Anderson, M. P., Grest, G. S. \& Sahni, P. S. (1984b). Computer simulation of grain growth - III. Influence of a particle dispersion. Acta Metallurgica, Vol.32, No. 9, pp. 1429-1438.

Srolovitz, D. J., Grest, G. S. \& Anderson, M. P. (1985). Computer simulation of grain growth - V. Abnormal grain growth. Acta Metallurgica, Vol. 33, No. 12, pp. 2233-2247.

Srolovitz, D. J., Grest, G. S. \& Anderson, M. P. (1986). Computer simulation of recrystallization - I. Homogeneous nucleation and growth. Acta Metallurgica, Vol. 34, No. 9, pp. 1833-1845.

Srolovitz, D. J., Grest, G. S., Anderson, M. P. \& Rollett, A. D. (1988). Computer simulation of recrystallization: II. Heterogenous nucleation and growth. Acta Metallurgica, Vol. 36, No. 8, pp. 2115-2128.

Tikare, V., Holm, E. A., Fan, D. \& Chen, L. Q. (1998). Comparison of phase-field and Potts models for coarsening processes. Acta Materialia, Vol. 47, No. 1, 363-371.

Von Neumann, J. (1952). Discussion: grain shapes and other metallurgical applications of topology. In: Metal Interfaces, ASM, Cleveland.

Walters, J., Kurtz, S., Wu, W.-T. \& Tang, J. (1997). The 'state of the art' in cold forming simulation. Journal of Materials Processing Technology, Vol. 71, No. 1, pp. 64-70.

Weiare, D. \& Kermode, J. P. (1983). Computer simulation of a two-dimensional soap froth: I. Method and motivation. Philosophical Magazine B, Vol. 48, No. 3, pp. 245-259.

Weiare, D. \& Kermode, J. P. (1984). Computer simulation of a two-dimensional soap froth: II. Analysis of results. Philosophical Magazine B, Vol. 50, No. 3, pp. 379-388.

Xiaoyan, S., Guoquan, L. \& Nanju, G. (2000). Re-analysis on grain size distribution during normal grain growth based on Monte Carlo simulation. Scripta Materialia, Vol. 43, pp. 355-359.

Yang, W., Chen, L.-Q. \& Messing, G. L. (1995). Computer simulation of anisotropic grain growth. Materials Science and Engineering A, Vol. 195, pp. 179-187.

Yang, Z., Sista, S., Elemer, J. W. \& DebRoy, T. (2000). Three dimensional Monte Carlo simulation of grain growth during GTA welding of titanium. Acta Materialia, Vol. 48, pp. 4813-4825. 
Yu, Q. \& Esche, S. K. (2003a). A Monte Carlo algorithm for single phase normal grain growth with improved accuracy and efficiency. Computational Materials Science, Vol. 27, No. 3, pp. 259-270.

$\mathrm{Yu}$, Q. \& Esche, S. K. (2003b). A new perspective on the normal grain growth exponent obtained in two-dimensional Monte Carlo simulations. Modelling and Simulation in Materials Science and Engineering, Vol. 11, No. 6, pp. 859-862.

$\mathrm{Yu}, \mathrm{Q}$. \& Esche, S. K. (2003c). Three-dimensional grain growth modeling with a Monte Carlo algorithm. Materials Letters, Vol. 57, No. 30, pp. 4622-4626.

$\mathrm{Yu}$, Q. \& Esche, S. K. (2004). Mesoscopic computer modeling of microstructure evolution within an object-oriented simulation framework. International Journal of Computational Engineering Science, Vol. 5, No. 3, pp. 451-469.

$\mathrm{Yu}, \mathrm{Q} ., \mathrm{Wu}, \mathrm{Y}$. \& Esche, S. K. (2005). Modeling of grain growth characteristics in threedimensional domains and two-dimensional cross sections. Metallurgical and Materials Transactions A, Vol. 36A, pp. 1661-1666.

$\mathrm{Yu}$, Q. \& Esche, S. K. (2005). A Multi-scale approach for microstructure prediction in thermo-mechanical processing of metals. Journal of Materials Processing Technology, Vol. 169, pp. 493-502. 


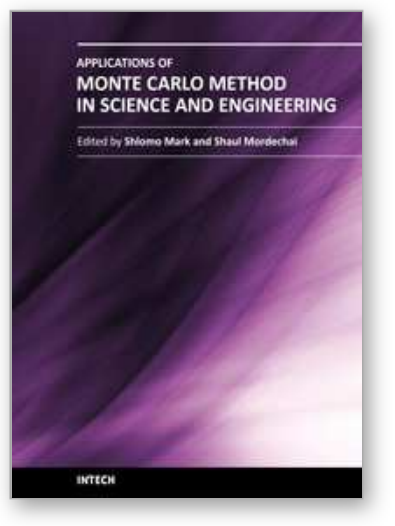

\section{Applications of Monte Carlo Method in Science and Engineering \\ Edited by Prof. Shaul Mordechai}

ISBN 978-953-307-691-1

Hard cover, 950 pages

Publisher InTech

Published online 28, February, 2011

Published in print edition February, 2011

In this book, Applications of Monte Carlo Method in Science and Engineering, we further expose the broad range of applications of Monte Carlo simulation in the fields of Quantum Physics, Statistical Physics, Reliability, Medical Physics, Polycrystalline Materials, Ising Model, Chemistry, Agriculture, Food Processing, X-ray Imaging, Electron Dynamics in Doped Semiconductors, Metallurgy, Remote Sensing and much more diverse topics. The book chapters included in this volume clearly reflect the current scientific importance of Monte Carlo techniques in various fields of research.

\section{How to reference}

In order to correctly reference this scholarly work, feel free to copy and paste the following:

Sven K. Esche (2011). Monte Carlo Simulations of Grain Growth in Metals, Applications of Monte Carlo Method in Science and Engineering, Prof. Shaul Mordechai (Ed.), ISBN: 978-953-307-691-1, InTech, Available from: http://www.intechopen.com/books/applications-of-monte-carlo-method-in-science-andengineering/monte-carlo-simulations-of-grain-growth-in-metals

\section{INTECH}

open science | open minds

\section{InTech Europe}

University Campus STeP Ri

Slavka Krautzeka 83/A

51000 Rijeka, Croatia

Phone: +385 (51) 770447

Fax: +385 (51) 686166

www.intechopen.com

\section{InTech China}

Unit 405, Office Block, Hotel Equatorial Shanghai

No.65, Yan An Road (West), Shanghai, 200040, China

中国上海市延安西路65号上海国际贵都大饭店办公楼 405 单元

Phone: +86-21-62489820

Fax: $+86-21-62489821$ 
(C) 2011 The Author(s). Licensee IntechOpen. This chapter is distributed under the terms of the Creative Commons Attribution-NonCommercialShareAlike-3.0 License, which permits use, distribution and reproduction for non-commercial purposes, provided the original is properly cited and derivative works building on this content are distributed under the same license. 